\title{
Deep Neural Network and Social Ski-Driver Optimization Algorithm for Power System Restoration with VSC - HVDC Technology
}

Jan Bhasha Shaik

A1 Global Institute of Engineering and Technology

Prakasam, Andhra Prades, India

janbhashashaik@gmail.com

\author{
Ganesh V \\ JNTUACEP \\ Pulivendula, Andhra Pradesh, India
}

\begin{abstract}
VSC-HVDC has to turn out to be a novel development for attaining a competent and consistent bulk power transmission. In the VSC-HVDC link, the main confronts is the "soft start-up" otherwise the power system restoration subsequent to the black-out system, against minimum response delay and over voltage. In this work, VSC-HVDC connection with Social Ski Driver algorithm- -trained Deep Neural Network (DNN), called as DNN- Social ski-Driver (SSD) algorithm, and it is proposed to reinstate the power rapidly. The experimentation is done in the system by producing blackout during the development of a three-phase fault. The evaluation performance is done in the VSC-HVDC with DNN-SSD in the conventional PI controller. The experimentation outcomes endow with more rapidly independent and restoration control for the reactive and real powers. The proposed algorithm reduces the over voltage while comparing it with the conventional algorithms.
\end{abstract}

Keywords: VSC-HVDC; Power System; PI Controller; Fault; DNN; Optimization Algorithm

\begin{tabular}{ll} 
Nomenclature & \\
\hline Abbreviations & Descriptions \\
\hline PI & Proportional Integral \\
HVDC & High Voltage Direct Current \\
VSWT & Variable Speed Wind Turbines \\
VSC & Voltage Source Converter \\
PFC & Primary Frequency Control \\
LCC & Line Commutated Converter \\
R-ECT & Rogowski coli based electronic Current Transformer \\
RMS & Root Mean Square \\
TW & Travelling Wave \\
MPC & Model Predictive Control \\
GEC & Generator Emulation Control \\
DCCB & Direct Breaker Circuit Breaker \\
GrHDP & Goal representation Heuristic Dynamic Programming \\
OWFs & Offshore Windfarms \\
SG & Synchronous Generator \\
EVCS & Enhanced Voltage Control Strategy \\
GWO & Grey Wolf Optimizer \\
WTGs & Wind Turbine Generators \\
SDC & Supplementary Damping Controller \\
D-CTW & Differential Current Travelling Wave \\
HDP & Heuristic Dynamic Programming \\
\hline
\end{tabular}

\section{Introduction}

Because of the full-controlled power electronic devices and voltage source converter, the VSC-HVDC application encompasses numerous methodological benefits like self-governing operate against the 
reactive and active power, low harmonics, the action not including $\mathrm{AC}$ grid sustaining, and maintenance polarity constant of the voltage while the reversal of the power flow [1]. Hence, it contains the significant value of realistic engineering in the distributed energy field such as extensive distance, interconnection, and massive-ability transmission of the power besides encompass received extensive concentration in the engineering field.

Since a new HVDC application, VSC based HVDC encompasses the benefits regarding independent control of reactive and active power, great the renewable energy incorporation regulation range. In China, by means of the speedy improvement and exploitation of VSC-HVDC, power system determination and develop to a comprehensive hybrid AC/DC power system in the prospect [19] [20], whereas inter-area oscillation is the major confronts for its safe and steady operation.

For the enormous incorporation of wind farms, VSC-HVDC associations are fast attractive to the main facilitators on the basis of the VSWT into power systems [1]. At the linking point of a VSC-HVDC, which is associated with a wind farm that has the ability to introduce wind power in an easier way than an instantly-associated with the wind farm, because of the effectual reactive and active power control emphasized using the stations in a converter manner. However, incorporation of extensive wind power plants encompass carried regarding numerous confronts that should be solved for a consistent recent power grids applications [3]. For example, weaken responses of the system determination of the consequence from the imbalances of power, therefore, reasoning frequency variations to be ahead of secure working restrictions. Extremely solid development, though, the development of appropriate methods was attained in the precedent concerning to facilitate the contribution of VSWT in the PFC [5]. Further lately, there an erstwhile investigation of active attempt on the incorporation of wind farms via the transmission of the VSC associations owing to the observed drawbacks that their interdependence might decrease the stability of frequency in the power system, this significant feature considered as the most important inspirations of this investigation of the study.

In the VSC-HVDC system to decrease fault experience, the cables of DC are extensively exploited in the transmission lines in untimely minimum VSC-HVDC voltage works. By means of the fast advancement of the DCCB and taking into consideration the clear benefits of the operating cost of transmission lines regarding cost expenditure and capacity of the transmission, VSC-HVDC grid on the basis of the DCCB and operating cost of the transmission lines determination and it turned out to be the major objectives of the HVDC advancement [6]. Nevertheless, faults occur in grounding which is a huge difficulty of VSC-HVDC with operating cost of transmission lines, since they happen additional often on operating cost of transmission lines. Set that the majority of the faults in grounding such as power supply, transient faults, which have the ability to rapidly renewed using modeling a reclosing of the sensible stratagem that has the ability to progress power supply consistency. Hence, it is essential to recognize precisely if the fault is transitory else enduring prior to the reclosing.

In [9] developed a new principle for fault location from the fault current signal on the basis of the large-frequency modules recognized. This algorithm has the ability to precisely recognize each fault line and categorize the fault conditions. In [10] developed a multiterminal TW fault position technique. It has the ability to rapidly, precisely, and consistently place the point of fault in the reduced acquisition of TW unit with the best possible position. In [11] presented a principle of time-domain fault-position appropriate for HVDC-TL. Moreover, it has the ability to place faults precisely on the HVDC lines yet in the scenario of signals loss from GPS. By exploiting measurements of the terminal, [12] paid the interest on faults in the DC line for the existing HVDC system of transmission with sections of cables and operating cost lines. It possesses the anti-interference capability for a description of examination needs in circuit breakers of HVDC. In [13], faults in HVDC of the multi-terminal power grid were analyzed. In [14] developed a 2-terminal technique TW on the basis of the fault position technique for HVDC systems in accordance with ground and aerial form TWs. In [15] developed on the precise fault position of the TW technique of operating cost of line-cable hybrid line and evaluated the manipulating reasons.

The main contribution of this paper is to present a VSC-HVDV connection, using DNN-SSD, to recover the power rapidly. Subsequent to the black-out, in VSC-HVDC DNN-SSD algorithm is developed to improve the capability of "Soft start-up". Furthermore, the proposed DNN-SSD algorithm performance is evaluated with the DNN-SSD with the PI controller.

\section{Literature Review}

In 2018, Luis M. Castroa and Enrique Acha [1] developed a novel technique to evaluate the involvement of VSC-HVDC, which was linked with wind farms of the power networks. Moreover, this was devised for facilitating the post-disturbance circumstances so as to evaluate the power system, and its frequency of the electrical, subsequent to the power shortcomings incidence; whereas a variety of VSC-HVDC linked 
with wind farms might concurrently experiment. Since resistant complete dynamic experimentation that might be consuming more time, the proposed technique resorts in order to model each and every power system modules by exploiting an improved steady-state power-flow design for the PFC time frame.

In 2020, Dong Wang, Mengqian Hou [2], addressed the issues in the fault position of transmission line for HVDC operating cost due to the VSC and LCC. There subsist two problems which ought to be addressed such as a) the fault position rules in TW that was the flexibility to converter structures and multi-terminal network topology b) the transfer technique for maximum frequency in TW signal. As a result, a new fault position rule in TW for the transmission line of the hybrid multi-terminal LCC-MMCHVDC was developed by reason of the R-ECT. Initially, for TW, the maximum frequency ability of the RECT was examined. Next, a new principle of fault position in TW on account of the secondary D-CTW was developed.

In 2020, Botong Li et al [3], developed an enduring fault recognition technique in the VSC-HVDC grid for single-pole grounding fault of operating cost of transmission lines due to the voltage in the fault line. Initially, the functioning principle of the proposed hybrid DCCB, and the emission procedure of the subjective capacitor of the proposed method was evaluated. Subsequently, for the voltage of the fault line, the transient procedures in the circumstances of the transient and permanent fault were evaluated subsequent to the isolation of the reclosing.

In 2017, Xudan Liu and Andreas Lindemann [4], proposed a GEC technique to present a VSC-HVDC converter station with synthetic inertia. Additionally, for VSC-HVDC linked OWFs, a synchronized control approach to attain improved capability for the allocation of synthetic inertia. Moreover, they had provided the rising renewable energy sources diffusion, which might cause an important minimization of the inertia system and determination. Therefore, consequence in a superior instability risk, comprehensive control loops to imitate both the characteristics of droop and the inertia of an SG.

In 2017, M. R. Safari Tirtashi and O. Samuelsson [5], worked on the small-disturbance electromechanical dynamics of a power system that can be interpreted to a corresponding circuit model with capacitances and inductances. Proportionate to local frequency, active power damping control by means of a VSC-HVDC connected otherwise storage of energy subsequently symbolizes establishing a resistor in the circuit design. This work exhibits which impedance corresponding have the ability to exploit for the selection for a value of the resistor, and consistently increase in the controller of damping which presents the utmost ratio of the damping. Additionally, it was exposed to how the idea was used without a circuit model.

In 2017, Yifei Guo et al [6], developed an EVCS on the basis of the MPC for VSC-HVDC connected OWFs. All wind farms, and wind WTGs part the VSC, were optimally integrated to manage voltages in the practicable series and decrease power losses in the system for the developed MPC based EVCS. Taking into consideration, a significant R/X OWF collector system ratio, the aspects in outputs of active power for WTGs on control of voltage were besides considered. The predictive technique of VSC with a usual cascaded control model was resultant in detail. By an analytical technique, the sensitivity coefficients were computed to enhance the computational effectiveness.

In 2018, Jose Luis Rodriguez-Amenedo et al [7], developed the parallel control operation for 2 VSCHVDC connections be integrating an OWF. Here, the main intention of this work was to suggest and confirm a control system which permits two VSC-HVDC parallel operation connections through dealing with the injected currents using the converters of the VSC. The setpoints of the currents were recognized through a controller of voltage to sustain constant frequency and voltage in the capacitor of the output filter.

In 2017, Yu Shen et al [8], developed an adaptive SDC for VSC-HVDC by exploiting the GrHDP. Evaluated with existing HDP, to produce an adaptive compensation signal, GrHDP augments a novel objective depiction network to attain further mapping association among the control action and the system states. In addition, the developed GrHDP-SDC was a design-free control method that does not necessitate the theoretical power system design and encompass rapid online learning capability to get used to the deviation of operating system circumstances.

\section{Controlling Principles and VSC-HVDC Link}

\subsection{VSC-HVDC Link Modeling}

The waveforms in association with the voltage of the inverter and rectifier are allotted to be sinusoidal additionally; the harmonics are wholly neglected in the steady-state investigation. Consider the dc magnitude of the voltages at the terminals of the inverter is $V_{i}$ and the rectifier is $V_{r}$ subsequently,

$$
\mathrm{V}_{\mathrm{r}}=\mathrm{t}_{\mathrm{r}} \mathrm{U}_{\mathrm{r}} \cos \beta_{\mathrm{r}}-\mathrm{R}_{\mathrm{r}} \mathrm{I}_{\mathrm{d}}
$$




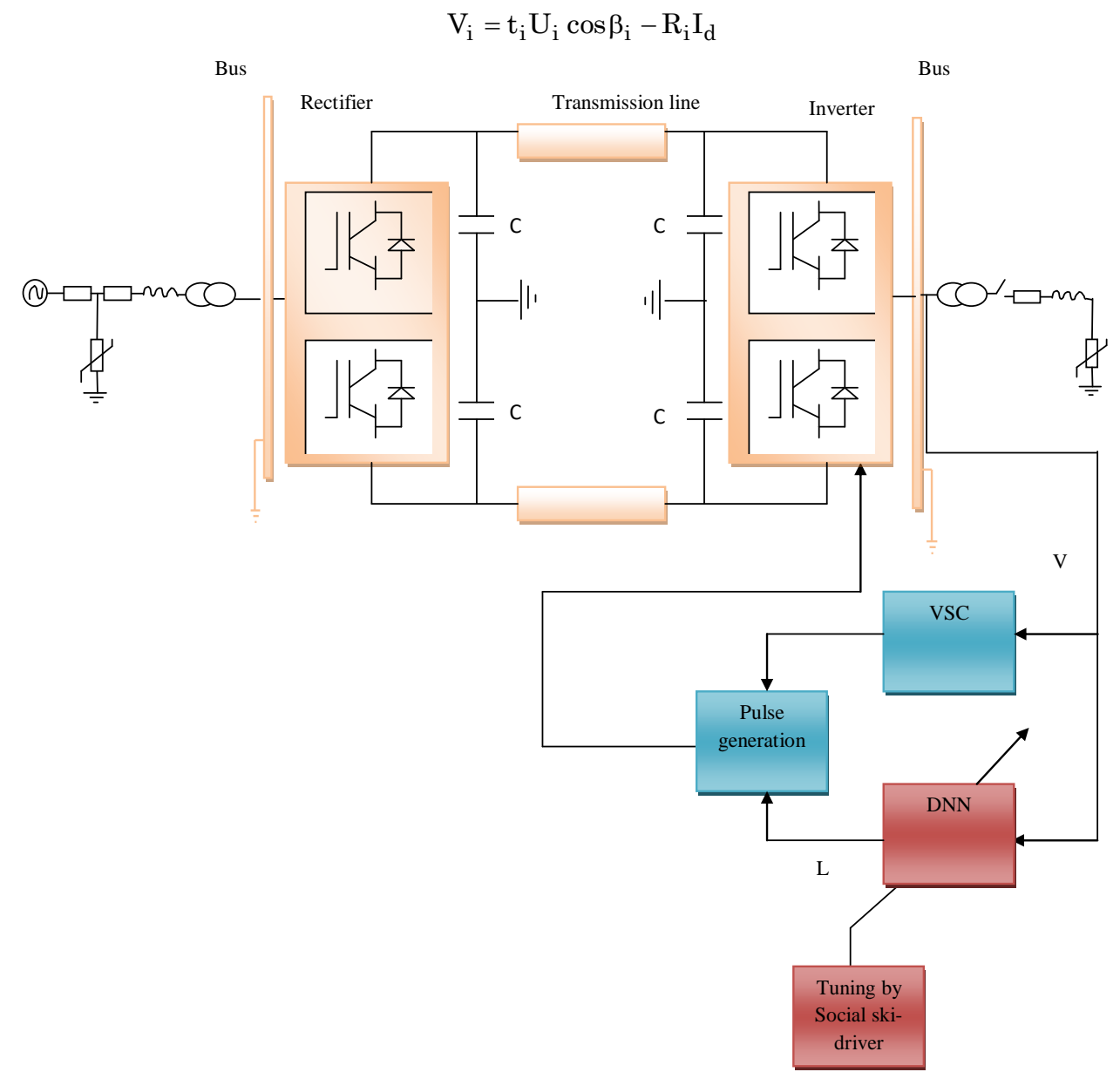

Fig. 1. Design of the DNN-SSD-based restoration exploiting VSC-HDVC connection

Consider $\mathrm{L}_{\mathrm{r}}, \mathrm{L}_{\mathrm{i}}, \mathrm{M}_{\mathrm{r}}, \mathrm{M}_{\mathrm{i}}$ be usually indicated as $\mathrm{L}$ and $\mathrm{M}$, that are the flows of the reactive and the active power into the dc network beside the terminals inverter side and the rectifier and it is described as follows:

$$
\begin{aligned}
& \mathrm{L}_{\mathrm{r}}=\mathrm{V}_{\mathrm{r}} \mathrm{I}_{\mathrm{d}} \\
& \mathrm{L}_{\mathrm{i}}=\mathrm{V}_{\mathrm{i}} \mathrm{I}_{\mathrm{d}} \\
& \mathrm{M}_{\mathrm{r}}=\mathrm{L}_{\mathrm{r}} \tan \psi_{\mathrm{r}} \\
& \mathrm{M}_{\mathrm{i}}=\mathrm{L}_{\mathrm{i}} \tan \psi_{\mathrm{i}} \\
& \tan \psi_{\mathrm{r}}=\frac{2 \eta_{\mathrm{r}}+\sin 2 \beta_{\mathrm{r}}-\sin 2\left(\beta_{\mathrm{r}}+\eta_{\mathrm{r}}\right)}{\cos 2 \beta_{\mathrm{r}}-\cos 2\left(\beta_{\mathrm{r}}+\eta_{\mathrm{r}}\right)} \\
& \tan \psi_{\mathrm{i}}=\frac{2 \eta_{\mathrm{i}}+\sin 2 \beta_{\mathrm{i}}-\sin 2\left(\beta_{\mathrm{i}}+\eta_{\mathrm{i}}\right)}{\cos 2 \beta_{\mathrm{i}}-\cos 2\left(\beta_{\mathrm{i}}+\eta_{\mathrm{i}}\right)}
\end{aligned}
$$

In eq. (7) and (8), $\beta_{\mathrm{i}}$ indicates the extinction angle for the inverter operation and $\beta_{\mathrm{r}}$ indicates the ignition angle for the operation of the rectifier. Moreover, $\delta$ indicates the line angle -to-neutral source voltage and $\xi$ indicates the angle of the basic line current. Hence, in eq. (5) and eq. (6) $\psi_{\mathrm{r}}$ and $\psi_{\mathrm{i}}$ indicates angles through the angle $\xi$ pauses angle $\delta . t_{\mathrm{r}}$ and $\mathrm{t}_{\mathrm{i}}$ indicates converter transformer tap settings, $R_{r}$ and $R_{i}$ indicates commutation resistances, $U_{r}$ and $U_{i}$ indicates on the AC terminals side for inverter and rectifier effectual phase voltage magnitudes. Moreover, $I_{d}$ indicates the HDVC connection dc current.

During the commutation phase, the phase currents cannot alter instantly. Hence, communication time is a number of times that is necessary for the transportation of the current, which transfers from one phase to another. The equivalent of the commutation time angle is indicated as $\eta$. For both inverter and rectifier sides, the overlap angle are calculated that is indicated as follows:

$$
\mu_{\mathrm{r}}=\cos ^{-1}\left(\cos \beta_{\mathrm{r}}-\frac{2 \mathrm{R}_{\mathrm{r}} \mathrm{I}_{\mathrm{d}}}{\mathrm{t}_{\mathrm{r}} \mathrm{U}_{\mathrm{r}}}\right)-\beta_{\mathrm{r}}
$$




$$
\mu_{\mathrm{i}}=\cos ^{-1}\left(\cos \beta_{\mathrm{i}}-\frac{2 \mathrm{R}_{\mathrm{i}} \mathrm{I}_{\mathrm{d}}}{\mathrm{t}_{\mathrm{i}} \mathrm{U}_{\mathrm{i}}}\right)-\beta_{\mathrm{i}}
$$

In the dc connection, the current between both the terminals is represented as $I_{d}$ which is indicated in eq. (11), whereas Y represents the HVDC resistance connection cable.

$$
\mathrm{I}_{\mathrm{d}}=\frac{\mathrm{V}_{\mathrm{r}}-\mathrm{V}_{\mathrm{i}}}{\mathrm{Y}}
$$

\subsection{Controlling Approaches}

For the three phases of VSC-HVDC, the control scheme is represented as the amalgamation of two independent controllers such as a) the controller of the current, produces switching signal which symbolizes signal of the reference and the measured signal and b) DC voltage controller, which provides the signals of the reference to the control system.

Since currents are transformed into the synchronous reference frame in the current controller, the signals of the DC in balanced sinusoidal circumstances, and absolute synchronization, are produced. Additionally, by exploiting the first order and second-order transfer function, the error function is decided to increase control against the external voltage of DC. In load variations, the feed-forward DC connection controller provides tremendous minimization. When exploiting the feed-forward model, a great increase in the controller of voltage by means of minimized error is not essential. The controller of DC link voltage for eternity controls the capacitor current; consequently that it upholds a better balance of the power.

\section{In the VSC-HVDC Connection by DNN-SSD-Restoration}

Fig 1 demonstrates the model of the DNN-SSD in the VSC-HVDC connection for power system restoration. By providing the VSC-HVDC links the restoration model outcomes, in fast system reinstatement. In this model, V can be indicated as input and $\mathrm{L}$ indicate as an NN output pulse. The output, and input, encompass a well-built persuade based on the quality in addition to the power stability. The obtained data is given to a learning process, consequently that the NN model [16] is recognized.

The method of restoration of the VSC-HVDC connection on the basis of the DNN has the subsequent benefits, initially; the multiple processing layers of DNN can efficiently extract representations of the time series. Subsequently, the tracking index on the basis of the DNN can reveal nonlinear relations. Hence, this work exploits the DNN to track the index, and after that decide the weight.

\subsection{Deep Neural Network}

A Deep Neural Network (DNN) is a neural network with a certain level of complexity, a neural network with more than two layers. DNN exploit sophisticated mathematical modelling to process data in complex ways [22]. Without loss of generalization, this work explains the basic principle of the DNN and the measurement of equivalent index weight on the basis of the DNN model as demonstrated in Fig 2. The output of the hidden layer is $G=\left[g_{1}, g_{2}, \ldots, g_{n}\right]$, whereas $g_{j}(j=1,2, \ldots ., m)$ indicates the output of the $j^{\text {th }}$ hidden unit. If the output of the output layer is $\mathrm{Z}_{\mathrm{k}}, \gamma_{1}$ and $\gamma_{2}$ represent the equivalent activation functions in the output and the hidden layers, correspondingly and it is represented as below:

$$
\begin{aligned}
& \mathrm{o}_{\mathrm{j}}=\sum_{\mathrm{i}=1}^{\mathrm{n}} \mathrm{y}_{\mathrm{i}} \mathrm{w}_{\mathrm{i}, \mathrm{j}}, \mathrm{g}_{\mathrm{j}}=\gamma_{1}\left(\mathrm{o}_{\mathrm{j}}\right) \\
& \mathrm{o}_{\mathrm{k}}=\sum_{\mathrm{i}=1}^{\mathrm{n}} \mathrm{y}_{\mathrm{i}} \mathrm{u}_{\mathrm{j}, \mathrm{k}}, \mathrm{z}_{\mathrm{k}}=\gamma_{1}\left(\mathrm{o}_{\mathrm{k}}\right)
\end{aligned}
$$

It ought to be illustrious which nonlinear activation function can comprehend the nonlinear transformation of the weighted data. Consequently, in eq. (12) and (13), nonlinear functions $\gamma_{1}$ and $\gamma_{2}$ can be a sign of the nonlinear interaction association amid different function and nonlinear influence of index.

The vector expression of eq. (12) and (13) are indicated in eq. (14) and eq. (15):

$$
\begin{aligned}
& \mathrm{G}=\gamma_{1}\left(\mathrm{YW}+\mathrm{a}_{1}\right) \\
& \mathrm{Z}_{\mathrm{k}}=\gamma_{2}\left(\mathrm{GU}+\mathrm{a}_{2}\right)
\end{aligned}
$$

Whereas 


$$
\begin{aligned}
\mathrm{W} & =\left[\begin{array}{cccc}
\mathrm{w}_{11} & \mathrm{w}_{12} & \cdots & \mathrm{w}_{1 \mathrm{~m}} \\
\mathrm{w}_{21} & \mathrm{w}_{22} & \cdots & \mathrm{w}_{2 \mathrm{~m}} \\
\vdots & \vdots & \vdots & \vdots \\
\mathrm{w}_{\mathrm{n} 1} & \mathrm{w}_{\mathrm{n} 2} & \cdots & \mathrm{w}_{\mathrm{nm}}
\end{array}\right] \\
\mathrm{U} & =\left[\begin{array}{c}
\mathrm{u}_{11} \\
\mathrm{u}_{21} \\
\vdots \\
\mathrm{u}_{\mathrm{n} 1}
\end{array}\right]
\end{aligned}
$$

By using the optimization model of eq. (18), the parameters of $\mathrm{NN}$ can be attained.

$$
\min \left[\mathrm{Z}_{\mathrm{k}}-\hat{\mathrm{Z}}_{\mathrm{k}}(\mathrm{Y})\right]+\lambda \mathrm{J}(\theta)
$$

In eq. (18), $\mathrm{J}(\theta)=\frac{1}{2}\left(|| \mathrm{W}||_{2}^{2}+|| \mathrm{U}||_{2}^{2}\right)$ is the regularization phrase. By the DNN, the weight matrixes $\mathrm{W}$ and $\mathrm{U}$ are trained, which reflect the connection amid different units in different layers of the NN.

Hence, this work presents a technique to compute the weights of the input unit in the direction of the output unit to detain the direct connection amid the input factor and output factor. Specifically:

$$
\begin{aligned}
& \frac{\partial \mathrm{Z}_{\mathrm{k}}}{\partial \mathrm{y}_{\mathrm{i}}}=\gamma_{2}(\mathrm{GU})^{\prime} \gamma_{1}(\mathrm{YW})^{\prime} \mathrm{U} \frac{\partial(\mathrm{YW})}{\partial \mathrm{y}_{\mathrm{i}}} \\
& \frac{\partial \mathrm{Z}_{\mathrm{k}}}{\partial \mathrm{y}_{\mathrm{i}}}=\gamma_{2}(\mathrm{GU})^{\prime} \gamma_{1}(\mathrm{YW})^{\prime} \mathrm{UW}_{\mathrm{i}}
\end{aligned}
$$

In eq. (20), $\mathrm{W}_{\mathrm{i}}=\left[\mathrm{w}_{\mathrm{i} 1}, \mathrm{y}_{\mathrm{i} 2}, \ldots, \mathrm{y}_{\mathrm{im}}\right],(\mathrm{i}=1,2, \ldots, \mathrm{n})$. It is significant to signify that $\gamma_{2}(\mathrm{GU})^{\prime}=1$ and $\gamma_{1}(\mathrm{YW})^{\prime}=1$ whilst the activation functions $\gamma_{1}$ and $\gamma_{2}$ indicates linear activation functions. In this scenario, $\frac{\partial \mathrm{Z}_{\mathrm{k}}}{\partial \mathrm{y}_{\mathrm{i}}}$ indicates a time-independent constant. If $\gamma_{1}$ and $\gamma_{2}$ indicates nonlinear activation functions, $\frac{\partial \mathrm{Z}_{\mathrm{k}}}{\partial \mathrm{y}_{\mathrm{it}}}$ of a different time, it might differ that is frequently abandoned in previous work.

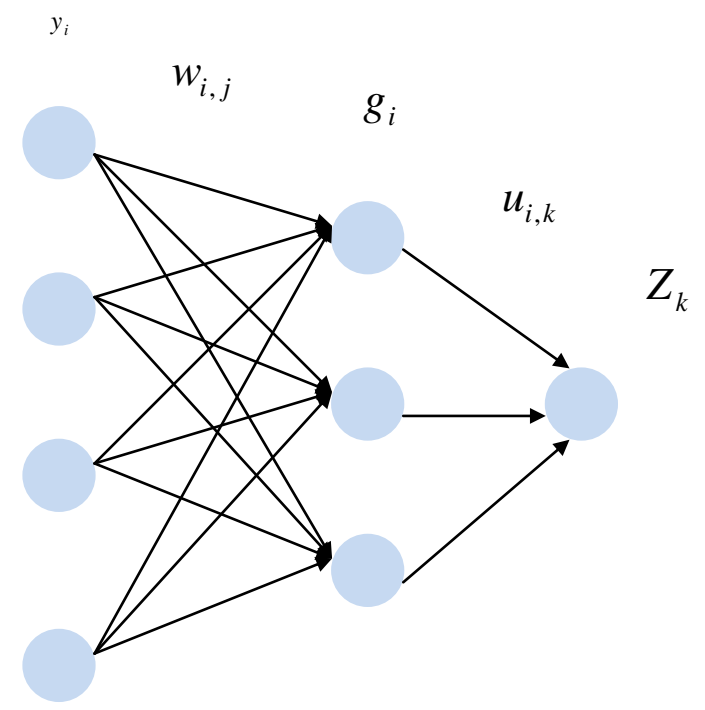

Fig. 2. Deep Neural Network model

\subsection{SSD Optimization Algorithm}

In this paper, a new optimization technique is referred to as Social Ski-Driver (SSD) approach [21].

From numerous different evolutionary optimization approaches, the behavior of SSD was enthused. Its name compliments to the actuality which its stochastic exploration in some way looks like the paths which ski-drivers get downhill. The proposed model encompasses numerous parameters; a concise explanation of these parameters is stated as follows:

* Locations of the agents $\left(Y_{i} \in R^{n}\right)$ : Agent locations are exploited to compute the intention model at the position, whereas $\mathrm{n}$ indicates the search space dimension.

* Preceding optimal location $\mathrm{Pt}_{\mathrm{i}}$ : For all agents, the fitness value is computed by exploiting the fitness model. For each agent, the fitness value is subsequently compared with its current location, and the optimal location is saved. This resembles the PSO approach [17]. 
* Mean global solution $\mathrm{Mg}_{\mathrm{i}}$ : In the SSD approach, since in the GWO [18]. Here, toward the global point the agent's travel that indicates the optimal 3 solutions mean.

$$
\mathrm{Mg}_{\mathrm{i}}^{\mathrm{t}}=\frac{\mathrm{Y}_{\alpha}+\mathrm{Y}_{\beta}+\mathrm{Y}_{\gamma}}{3}
$$

In eq. (21), $\mathrm{Y}_{\alpha}+\mathrm{Y}_{\beta}+\mathrm{Y}_{\gamma}$ indicates the optimal 3 solutions.

* Agents velocity $\left(\mathrm{U}_{\mathrm{i}}\right)$ : By adding velocity $\mathrm{U}_{\mathrm{i}}$ the location of the agents' is updated as below:

$$
\mathrm{Y}_{\mathrm{i}}^{\mathrm{t}+1}=\mathrm{Y}_{\mathrm{i}}^{\mathrm{t}}+\mathrm{U}_{\mathrm{i}}^{\mathrm{t}}
$$

where as,

$$
\mathrm{U}_{\mathrm{i}}^{\mathrm{t}+1}= \begin{cases}\mathrm{c} \sin \left(\mathrm{r}_{1}\right)\left(\mathrm{Pt}_{\mathrm{i}}^{\mathrm{t}}-\mathrm{Y}_{\mathrm{i}}^{\mathrm{t}}\right)+\sin \left(\mathrm{r}_{1}\right)\left(\mathrm{Mg}_{\mathrm{i}}^{\mathrm{t}}-\mathrm{Y}_{\mathrm{i}}^{\mathrm{t}}\right) & \text { if } \mathrm{r}_{2} \leq 0.5 \\ \mathrm{c} \sin \left(\mathrm{r}_{1}\right)\left(\mathrm{Pt}_{\mathrm{i}}^{\mathrm{t}}-\mathrm{Y}_{\mathrm{i}}^{\mathrm{t}}\right)+\sin \left(\mathrm{r}_{1}\right)\left(\mathrm{Mg}_{\mathrm{i}}^{\mathrm{t}}-\mathrm{Y}_{\mathrm{i}}^{\mathrm{t}}\right) & \text { if } \mathrm{r}_{2}>0.5\end{cases}
$$

In eq. (22), $\mathrm{U}_{\mathrm{i}}$ indicates the velocity of $\mathrm{Y}_{\mathrm{i}}, \mathrm{Pt}_{\mathrm{i}}$ indicates the optimal solution of the $\mathrm{i}^{\mathrm{th}}$ agent, $\mathrm{r}_{1}$ and $\mathrm{r}_{2}$ indicates uniformly produced arbitrary numbers in the range of $[0,1]$. For the complete population, $\mathrm{Mg}_{\mathrm{i}}$ indicates the mean global solution, and $\mathrm{c}$ indicates a parameter that is exploited to construct a balance among exploitation and the exploration which is computed as below:

$c^{t+1}=\beta c^{t}$, whereas $t$ indicates the current iteration and $0<\beta<1$ is exploited to minimize the $c$ value. Therefore, $c \rightarrow 0$, whereas $t \rightarrow t_{\max }$ and $t_{\max }$ indicates the utmost count of iterations.

As stated in eq. (23), for the agents, the moving directions are not uncomplicated as in PSO and GWO, and it is owing to the sine and cosine functions. Moreover, in the SSD approach, easy instances of two agents are altered. This states the proposed approach an improved-directed exploration capability and creates the directions of search to be diversified, however in a directed form.

For near-optimal or optimal solutions, the major aim of SSD is to explore in the space. The number of factors that are required to be optimized will decides the space dimension. The locations $\left(\mathrm{Y}_{\mathrm{i}}\right)$ of agents in SSD are arbitrarily initialized, whereas the user decides the number of agents. The agents' location is updated on their own. Additionally, previous locations' velocity is stated in eq. (22). As well, the velocities of the agents are arbitrarily initialized, and it is modified along with eq. (23). As stated in eq. (23), the attuned agent's velocity is based upon the distance among the current location, $\mathrm{Y}_{\mathrm{t}}^{\mathrm{i}}$, and the previous optimal location $\mathrm{pt}_{\mathrm{i}}$, the distance among the current location, $\mathrm{Y}_{\mathrm{t}}^{\mathrm{i}}$, and the $M g_{i}$ indicates the mean global solution.

Therefore, in SSD, the agents move toward the mean of the optimal three solutions that creates an SSD approach that is more common than PSO. In SSD, the agents are altered not in a simple way that presents the SSD approach enhanced exploration abilities. The SSD steps are stated as follows:

Pseudo code of the SSD approach

Initializes the agents' velocities $\mathrm{U}_{\mathrm{i}}$ and locations $\mathrm{Y}$, Consider the fitness model is low

While the terminate condition is not attained do

For all agents do

Compute fitness value

Based on their fitness value sort the agents

Compute the mean global solution and the previous optimal

location

As stated in eq. (22), by updating the agent's locations

produced new solutions

As stated in eq. (23), alter the velocity of the agents

End while

End for

Return the optimal solution

\section{Results and Discussions}

\subsection{Experimental Procedure}

The experimentation procedure of the DNN-SSD-based VSC-HVDC connection is attained by exploiting MATLAB. The common procedure of VSC-HVDC is attained from "http://in.mathworks.com/help/physmod/sps/powersys/ug/vsc-based-HVDC-link.html". 


\subsection{Comparative Analysis}

The comparative analysis of RMS voltage of Neural Network (NN) based VSC-HVDC [16], common VSCHVDC, Artificial Neural Network (ANN)-Levenberg Marquardt (LM) [23], and Deep Neural Network (DNN) [22] -SSD-based VSC-HVDC is demonstrated in Fig 3. Generally, RMS represents the determination of the divergence among the actual and predicted value that is attained from testing. The voltage of RMS is attained for three phases like phase A, B and C. At each phase, the value of the RMS for the developed DNN-SSD-based controller is lesser than the existing models. Therefore, on taking into consideration of the proposed method, the slightest RMS value is attained with the conventional model. In addition, the proposed model is $12 \%$ better than the PI, $13 \%$ better than the NN, $21 \%$ superior to the ANN-LM-based controller for phase A. The overall analysis shows that the proposed DNN-SSD-based VSC-HVDC exhibits superior restoration for the power system than the other conentional methods.

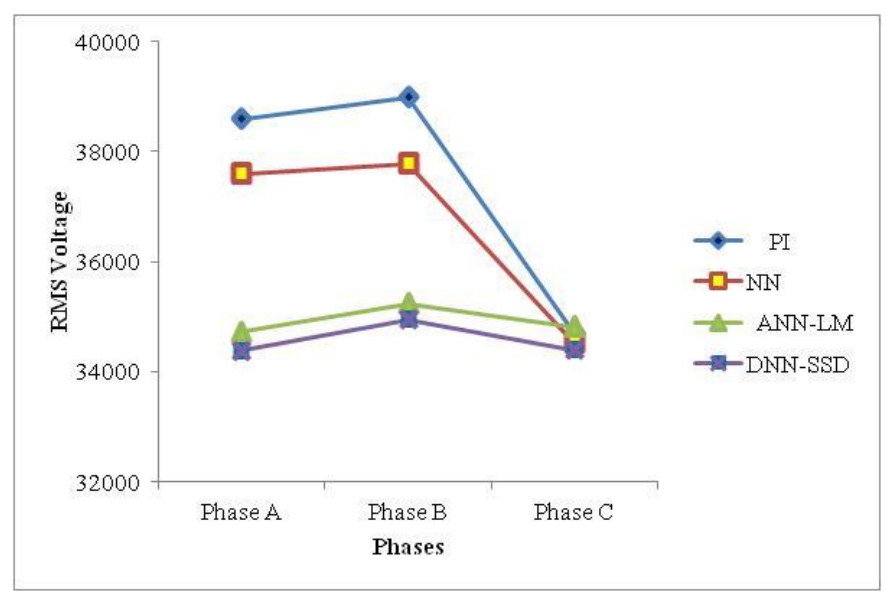

Fig. 3. Comparative analysis of the RMS voltage for several phase variations

\section{Conclusion}

The DNN-SSD based, the VSC-HVDC system encompass turn out to be economically and technically, possible for the power system restoration, subsequent to black-out. Hence, this work has proposed a novel VSC-HVDC technology, derived from DNN-SSD, to reinstate the power rapidly. Moreover, the consequences had revealed that against voltages are minimized in the restoration of the power system procedure, by means of minimum delay in response. DNN-SSD method encompasses enhanced "Soft start-up" ability of VSC-HVDC, subsequent to black-out. The overall performances of the proposed DNN SSD and DNN-SSD controllers were evaluated with the PI controller.

\section{Compliance with Ethical Standards}

Conflicts of interest: Authors declared that they have no conflict of interest.

Human participants: The conducted research follows the ethical standards and the authors ensured that they have not conducted any studies with human participants or animals.

\section{References}

[1] Luis M. Castro, Enrique Acha,"A new method to assess the contribution of VSC-HVDC connected wind farms to the primary frequency control of power networks", Electric Power Systems Research, Volume 154, January 2018, Pages 48-58.

[2] Dong Wang, Mengqian Hou,"Travelling wave fault location principle for hybrid multi-terminal LCC-VSC-HVDC transmission line based on R-ECT", International Journal of Electrical Power \& Energy Systems, Volume 117, May 2020.

[3] Botong Li, Hanqing Cui, Bin Li, Weijie Wen, Dongkang Dai,"A permanent fault identification method for singlepole grounding fault of overhead transmission lines in VSC-HVDC grid based on fault line voltage",International Journal of Electrical Power \& Energy Systems, Volume 117, May 2020.

[4] X. Liu and A. Lindemann, "Control of VSC-HVDC Connected Offshore Windfarms for Providing Synthetic Inertia," IEEE Journal of Emerging and Selected Topics in Power Electronics, Volume. 6, Issue. 3, page no. 1407-1417, September. 2018. 
[5] M. R. S. Tirtashi, O. Samuelsson and J. Svensson, "Impedance Matching for VSC-HVDC and Energy Storage Damping Controllers," IEEE Transactions on Power Delivery, Volume. 33, Issue. 2, page no. 1016-1017, April 2018.

[6] Y. Guo, H. Gao, Q. Wu, H. Zhao, J. Østergaard and M. Shahidehpour, "Enhanced Voltage Control of VSC-HVDCConnected Offshore Wind Farms Based on Model Predictive Control," IEEE Transactions on Sustainable Energy, Volume. 9, Issue. 1, page no. 474-487, January 2018.

[7] J. L. Rodríguez-Amenedo, S. Arnaltes-Gómez, M. Aragüés-Peñalba and O. Gomis-Bellmunt, "Control of the Parallel Operation of VSC-HVDC Links Connected to an Offshore Wind Farm," IEEE Transactions on Power Delivery, Volume. 34, Issue. 1, page no. 32-41, Feburary. 2019.

[8] Y. Shen, W. Yao, J. Wen, H. He and W. Chen, "Adaptive Supplementary Damping Control of VSC-HVDC for Interarea Oscillation Using GrHDP," IEEE Transactions on Power Systems, Volume. 33, Issue. 2, page no. 1777 1789, March 2018.

[9] Li JW, Yang QQ, Mu H, Blond SL, He HW. A new fault detection and fault location method for multi-terminal high voltage direct current of offshore wind farm. Appl Energy issue 220, Volume.13, -20, 2018.

[10] Deng F, Zeng XJ, Pan LL. Research on multi-terminal traveling wave fault location method in complicated networks based on cloud computing platform. Protect Control Mod Power Syst; Volume no 2(2), page no 199$210,2017$.

[11] Liang YS, Wang G, Li HF. Time-domain fault-location method on HVDC transmission lines under unsynchronized two-end measurement and uncertain line parameters. IEEE Trans Power Delivery; Volume 30(3), page no 1031-8, 2015.

[12] Nanayakkara OMKK, Rajapakse AD, Wachal R. Location of DC line faults in conventional HVDC systems with segments of cables and overhead lines using terminal measurements. IEEE Trans Power Delivery; Volume no 27(1), 2012.

[13] Belda NA, Plet CA, Smeets RPP. Analysis of faults in multiterminal HVDC grid for definition of test requirements of HVDC circuit breakers. IEEE Trans Power Delivery, Volume no 33(1), page no 403-11, 2018.

[14] Fernandes PC, Naomira Gomes Venzi Gonçalves H, Melo e Silva K, Vigolvino Lopes F. Two-terminal modal traveling wave-based fault location method for HVDC systems. In: Workshop on Communication Networks and Power Systems (WCNPS); page no 1-4, 2018.

[15] Xun L, Shungui L, Ronghui H, Jingwen A, Yunzhu A, Ping C, et al. Study on accuracy traveling wave fault location method of overhead line cable hybrid line and its influencing factors. 2017 Chinese Automation Congress (CAC) page no 4593-7, 2017.

[16] E. Nishani and B. Çiço, "Computer vision approaches based on deep learning and neural networks: Deep neural networks for video analysis of human pose estimation," 2017 6th Mediterranean Conference on Embedded Computing (MECO), Bar, page no 1-4, 2017.

[17] Poli R, Kennedy J, Blackwell T, "Particle swarm optimization. Swarm Intell 1(1)", page no. 33-57, 2007.

[18] Mirjalili S, Mirjalili SM, Lewis A," Grey wolf optimizer", Adv Eng Softw 69, page no. 46-61 2014.

[19] SK.Mahammad Shareef and Dr.R.Srinivasa Rao,"A Hybrid Learning Algorithm for Optimal Reactive Power Dispatch under Unbalanced Conditions",Journal of Computational Mechanics, Power System and Control (JCMPS), Volume 1, Issue 1, October 2018.

[20] Subramanyam T C,Tulasi Ram S S,Subrahmanyam J B V,"An Adaptive Chicken Swarm Algorithm to Solve Optimal Power Flow Problem Considering FACTS Device",Journal of Computational Mechanics, Power System and Control (JCMPS),Volume 2, Issue 1, January 2019.

[21] Alaa Tharwat, Thomas Gabel, "Parameters optimization of support vector machines for imbalanced data using social ski driver algorithm" -Neural Computing and Applications, pp. 1-14, 2019.

[22] P. Yi, Z. Jianyong, Y. Yun, Z. Rui, Z. Cheng and S. Tian, "An Electricity Load Forecasting Approach Combining DBN-Based Deep Neural Network and NAR Model for the Integrated Energy Systems," 2019 IEEE International Conference on Big Data and Smart Computing (BigComp), Kyoto, Japan, 2019, pp. 1-4.

[23] W. Lin and G. Chen, "Large Memory Capacity in Chaotic Artificial Neural Networks: A View of the AntiIntegrable Limit," IEEE Transactions on Neural Networks, vol. 20, no. 8, pp. 1340-1351, Aug. 2009. 